\section{Lesión pulmonar aguda tras transfusión de concentrado de hematíes en el paciente pediátrico. Presentación de caso y revisión de la literatura}

Pérez-Caballero-Macarrón $C^{1}$, Moreno-Jiménez $\mathrm{G}^{2}$, Coca-Pérez $\mathrm{A}^{1}$, VázquezMartínez $\mathrm{JL}^{1}$, Tenorio-Núñez $\mathrm{M}^{2}$, Stanescu $\mathrm{S}^{1}$, Tapia-Moreno $\mathrm{R}^{1}$, JiménezMartín $\mathrm{A}^{2}$

\section{Resumen}

La lesión pulmonar aguda (LPA) relacionada con la transfusión TRALI (transfusion related acute lung injury) se define como una lesión pulmonar que se desarrolla durante o dentro de las primeras seis horas post-transfusionales, con una $\mathrm{PaO} 2 / \mathrm{FiO} 2$ igual o menor de $300 \mathrm{mmHg}$, en ausencia de edema pulmonar cardiogénico, o de otro factor de riesgo de LPA. Es una complicación subestimada en niños enfermos críticamente debido a que se asocia los síntomas a la enfermedad de base, siendo la causa principal de muerte relacionada con la transfusión. Presentamos un caso de TRALI grave en un niño durante la transfusión de un concentrado de hematíes, procedente de una donadora multípara, en una Unidad de Cuidados Intensivos Pediátricos, con resultado favorable. El tratamiento es de soporte en función de la gravedad del cuadro clínico y la prevención se centra en tres estrategias: selección de donantes, actuación sobre el almacenamiento de los productos hemáticos y evitar las transfusiones innecesarias. Es importante incluir casos pediátricos de TRALI en la literatura médica para avanzar en la comprensión de esta complicación transfusional en los niños.

PALABRAS CLAVE: lesión pulmonar aguda causada por transfusión (TRALI), transfusión sanguínea, reacción transfusional, pediatría.
${ }^{1}$ UCI Pediatría. Servicio de Pediatría.

${ }^{2}$ Servicio de Hematología.

Hospital Ramón y Cajal, Madrid, España.

Recibido: 5 de julio del 2016

Aceptado: 6 de marzo del 2017

Correspondencia

MD. PhD. César Pérez-Caballero Macarrón

cesarperezcaballero@yahoo.es

Este artículo debe citarse como

Pérez-Caballero-Macarrón C, Moreno-Jiménez G, Coca-Pérez A, Vázquez-Martínez JL, Tenorio-Núñez $\mathrm{M}$, Stanescu $\mathrm{S}$ et al. Lesión pulmonar aguda tras transfusión de concentrado de hematíes en el paciente pediátrico. Presentación de caso y revisión de la literatura. Acta Pediatr Mex. 2017;38(6):386-393. 


\section{Transfusion related acute lung injury in paediatric patients. Case report and review of the literature.}

Pérez-Caballero-Macarrón $C^{1}$, Moreno-Jiménez $\mathrm{G}^{2}$, Coca-Pérez $\mathrm{A}^{1}$, VázquezMartínez $\mathrm{JL}^{1}$, Tenorio-Núñez $\mathrm{M}^{2}$, Stanescu $\mathrm{S}^{1}$, Tapia-Moreno $\mathrm{R}^{1}$, JiménezMartín $\mathrm{A}^{2}$

\begin{abstract}
Transfusion related acute lung injury (TRALI) is characterized by the onset of acute noncardiogenic pulmonary oedema within the first six hours following transfusion of blood products. The overall incidence seems underestimated in critically ill children, considering that the symptoms could be overlapping with the underlying disease. It is a serious complication and it is considered the major cause of transfusionrelated death. The therapy is supportive and the prevention focuses in three major points: donor selection, the suitable storage of blood products and avoid unnecessary transfusions. We present a case of severe TRALI in a child following packed red blood cells transfusion from a multiparous woman donor. It is mandatory to report TRALI paediatric cases in order to understand the outcome in children.
\end{abstract}

KEYWORDS: transfusion related acute lung injury; blood transfusion complication; children

\author{
${ }^{1}$ UCI Pediatría. Servicio de Pediatría. \\ ${ }^{2}$ Servicio de Hematología. \\ Hospital Ramón y Cajal, Madrid, España. \\ Correspondence \\ MD. PhD. César Pérez-Caballero Macarrón \\ cesarperezcaballero@yahoo.es
}

\section{INTRODUCCIÓN}

La lesión pulmonar aguda (LPA) relacionada con la transfusión TRALI (transfusion related acute lung injury) ocurre durante o después de una transfusión de productos sanguíneos. ${ }^{1}$ Es una complicación subestimada con alta morbilidad y en los últimos cinco años se ha convertido en la causa principal de muerte relacionada con la transfusión. ${ }^{2,3}$

De acuerdo con la definición internacional, reconocida por la American-European Consensus Conference en $2004^{4}$, la TRALI se define como una LPA que se desarrolla durante o dentro de las primeras seis horas post-transfusionales, con una $\mathrm{PaO} 2 / \mathrm{FiO} 2$ igual o menor de $300 \mathrm{mmHg}$, en ausencia de edema pulmonar cardiogénico, o de otro factor de riesgo de LPA ${ }^{5}$. Aunque la definición establece seis horas como límite para el inicio de los síntomas, generalmente suele presentarse en las primeras dos horas después de la transfusión.

La TRALI se describió por primera vez en la década de los años 50 del siglo pasado; la primera serie de casos se publicó en $1966 .{ }^{6}$ La mayoría de los casos de TRALI se refieren a pacientes 
adultos. Se estima la frecuencia en la población pediátrica en una de cada 2.000 unidades transfusiones con una tasa de mortalidad de $15 \%^{7}$ cifras similares a lo publicado en adultos. Ésta se ha relacionado con todos los componentes sanguíneos, aunque con más frecuencia en los que contienen plasma, ${ }^{8}$ debido a la presencia de anticuerpos antileucocitarios (TRALI inmune). También puede producirse por la acción de sustancias solubles, como lípidos liberados por los hematíes y plaquetas almacenados (TRALI no inmune). Este ocurre sobre todo en pacientes con procesos hematoncológicos y enfermedades cardíacas, siendo esta reacción menos grave que en el TRALI inmune.

El diagnóstico es fundamentalmente clínico ante la presencia de un cuadro de hipoxemia aguda y edema pulmonar no cardiogénico, durante o después de una transfusión de productos sanguíneos. Ante la sospecha de una reacción de este tipo, se debe suspender la transfusión. El tratamiento de la TRALI no se diferencia del tratamiento de la LPA de cualquier otra etiología. Los casos leves pueden ser tratados con medidas convencionales de oxígeno; en los casos graves, soporte respiratorio con ventilación mecánica. ${ }^{9}$

Es importante incluir casos pediátricos de TRALI en la literatura médica para avanzar en la comprensión de esta complicación transfusional en los niños. Presentamos un caso de TRALI grave atendido en una Unidad de Cuidados Intensivos Pediátricos, con recuperación completa del paciente.

\section{CASO CLÍNICO}

Adolescente de 16 años de edad con diagnóstico de coartación aórtica severa corregida a los cuatro meses, en quien se realizó cateterismo cardiaco 48 horas antes de ingresar por anemización aguda con repercusión hemodinámica: taquicardia sinusal (FC: 120 l/m) e hipotensión arterial (95/50 mmHg). La auscultación pulmonar es normal, no hay distres respiratorio y saturación de O2 93 \%. Dolor a la palpación en flanco derecho, con defensa abdominal y Blumberg negativo. No hay hepatomegalia, plétora yugular, ni ritmo de galope que sugieran insuficiencia cardiaca.

$\mathrm{Al}$ ingreso, hemoglobina de $7 \mathrm{~g} / \mathrm{dL}$; leucocitos 8420/mm3 (72.9\% polimorfonucleares; $12.5 \%$ linfocitos) y $141.000 / \mathrm{mm} 3$ plaquetas. La coagulación, bioquímica, ácido láctico y gasometría sin alteraciones; con un péptido natriurético tipo B de 283.8 pg/mL. Una angioTac abdominal y pélvica mostró malformación de la vasculatura renal y hematoma retroperitoneal derecho sin signos de sangrado activo (Figura 1).

Se transfundieron dos concentrados de hematíes durante la cual aumentó el trabajo respiratorio con hipoxemia y saturación de O2 72\%. No hubo respuesta a oxigenoterapia de alto flujo y requirió ventilación mecánica. En la radiografía de tórax se observaron opacidades pulmonares bilaterales y difusas sin cardiomegalia (Figura 2), siendo la radiografía previa a la transfusión normal. El electrocardiograma y el ecocardiograma descartaron la posibilidad de miocardiopatía.

Ante la sospecha de TRALI basada en la relación temporal entre la clínica respiratoria y la transfusión con la presencia de infiltrados pulmonares bilaterales difusos y la falta de síntomas de sobrecarga circulatoria, se inició tratamiento esteroideo y se suspendió la transfusión (lo cual se comunicó al Servicio de Hematología) y se aplicó el protocolo de nuestro centro sobre eventos relacionados con la transfusión. El producto sanguíneo se regresó al banco de sangre para iniciar la investigación.

El paciente tuvo una mejoría respiratoria progresiva que permitió extubación programada 48 horas después. El angioTAC de control mostró 


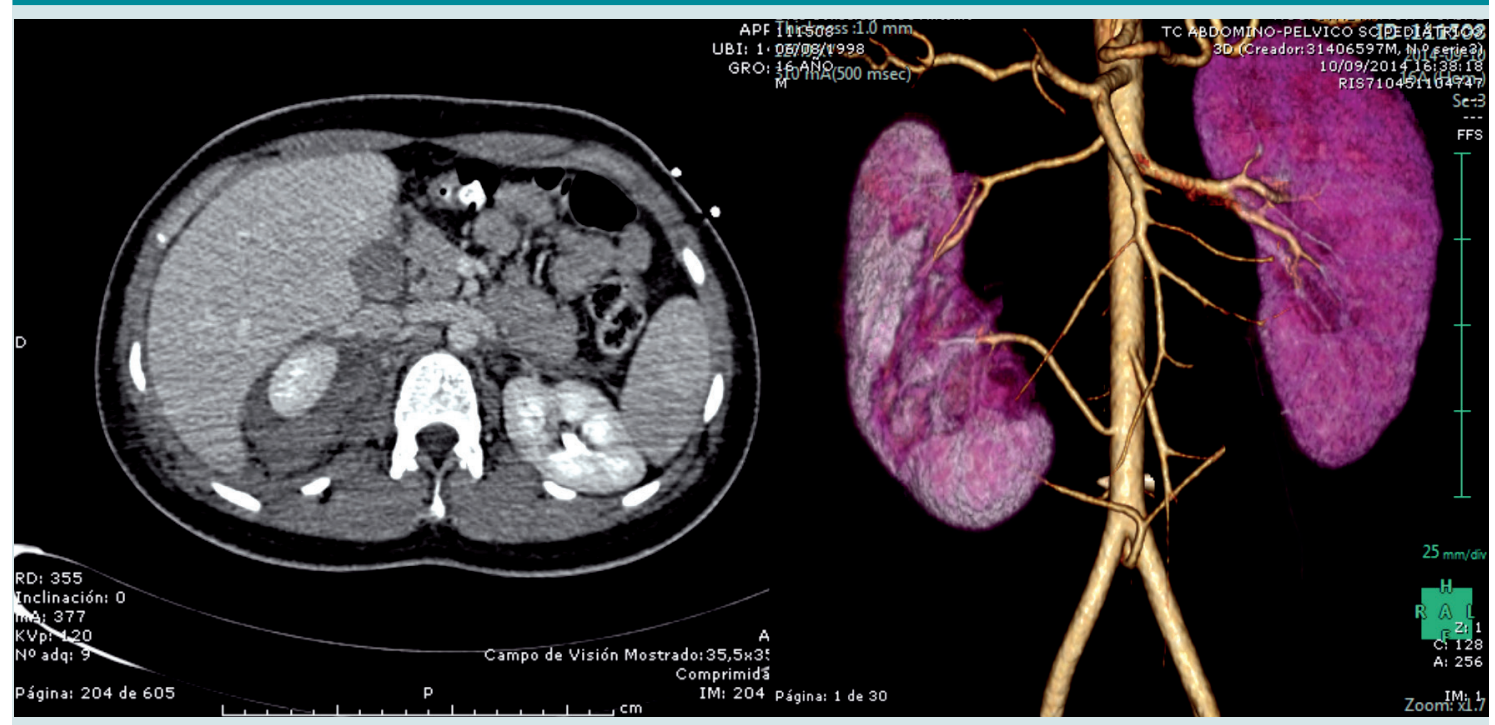

Figura 1. AngioTac abdómino-pélvico: hematoma retroperitoneal derecho sin signos de sangrado activo. Malformación de la vasculatura renal.

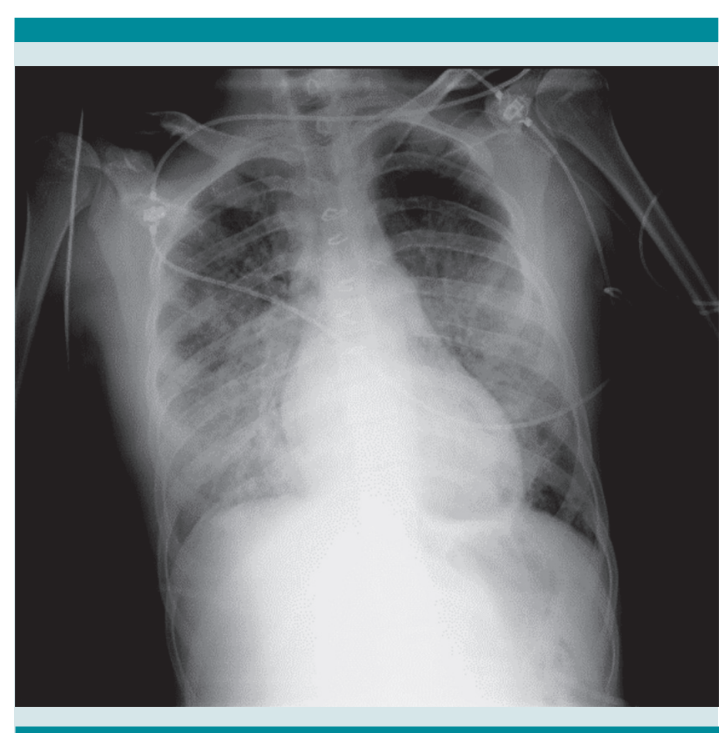

Figura 2. Radiografía de tórax tras suspender la transfusión: opacidades pulmonares bilaterales y difusas sin cardiomegalia.

leve disminución de tamaño del hematoma retroperitoneal derecho sin signos de sangrado activo; un patrón pulmonar con afectación de ambas bases y língula de posible origen inflamatorio. En la radiografía de tórax, realizada al cuarto día de hospitalización, desaparecieron las lesiones previas (Figura 3). No requirió nuevas transfusiones de productos sanguíneos y man-

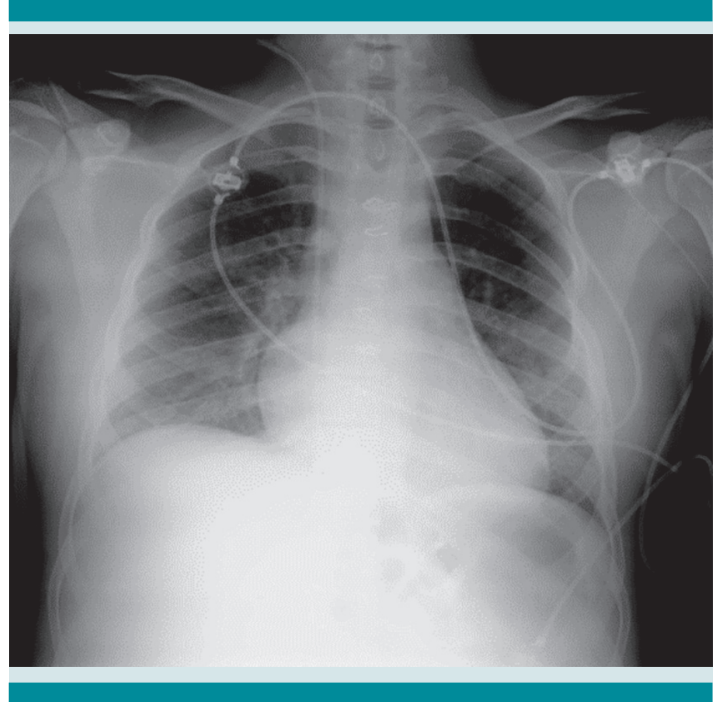

Figura 3. Radiografía de tórax tras extubación al cuarto día de ingreso en la UCIP. 
tuvo buena dinámica respiratoria sin necesitar oxígeno suplementario.

En el estudio realizado en el CRT-CAM, la detección de anticuerpos antineutrófilos, mediante citometría de flujo, fue positiva en una de las donadoras y la búsqueda de anticuerpos anti HLA frente a anticuerpos IgG/lgM anti-HLA clase I y clase II, fue negativa. Dado este hallazgo, la donadora fue excluida para futuras donaciones.

\section{DISCUSIÓN}

La TRALI es un síndrome clínico que se caracteriza por hipoxemia aguda y edema pulmonar no cardiogénico, que se presenta durante o dentro de las seis horas siguientes a la transfusión de productos sanguíneos. Es una entidad infra diagnosticada en la edad pediátrica y los estudios realizados son casos aislados. El grupo de hemovigilancia nacional del Reino Unido (SHOT), señaló que el $11 \%$ de los casos de TRALI ocurrían en niños entre 2 y 17 años de edad.?

Se ha propuesto una clasificación atendiendo al tiempo de aparición: TRALI clásica, que comienza durante las primeras $6 \mathrm{~h}$ de la transfusión y que coincidiría con el modelo de TRALI inmune y TRALI diferida o tardía, que comienza entre las 6 y las 72 h de la transfusión y cuyo mecanismo de producción sería por mediadores. ${ }^{10}$

La TRALI no se relaciona con la edad, sexo, ni se asocia con incompatibilidad de grupo entre donadores y receptores o número de transfusiones previas. La patogénesis de la TRALI no se conoce con exactitud; ;1 se han propuesto dos hipótesis no excluyentes: la primera sugiere que la TRALI es una reacción inmune causada por la interacción entre los leucocitos de los receptores y los anticuerpos antileucocitos de los donadores, induciendo el secuestro y activación de neutrófilos en los pulmones, produciendo daño endotelial y aumento de la permeabilidad vascular. ${ }^{12}$ Sin em- bargo, en algunos casos de TRALI no se identificó ningún anti HLA del donador ni anticuerpos antineutrófilos específicos que pudiesen explicar la presencia deTRALI. Por ello, se ha propuesto un segundo mecanismo: un modelo en "dos-fases", en el que en una primera fase dependiente de la condición del paciente (sepsis, cirugía reciente, lesión traumática, malignidad, transfusión masiva), se produce una activación del endotelio pulmonar y un secuestro de granulocitos en los pulmones. En una segunda fase, dependiente de la propia transfusión, se activarían los neutrófilos en el endotelio pulmonar, como resultado de los anticuerpos transfundidos $\mathrm{u}$ otros mediadores inflamatorios, lo que provocaría el daño capilar. Los anticuerpos involucrados en la TRALI estarían dirigidos tanto contra HLA como contra antígenos HNA expresados en los neutrófilos, pero los documentados con más frecuencia son los anticuerpos dirigidos contra HNA-1a, HNA1b, HNA-2a, HNA-3a (5b) y HLA-A2. ${ }^{13}$

La TRALI es un diagnóstico clínico; no existe una prueba de laboratorio específica, dependiendo por tanto de la exclusión de otras posibles causas de LPA. Nuestra paciente cumplía los criterios de TRALI proporcionados por el Grupo de Consenso Canadiense $2004,{ }^{14}$ no tuvo síntomas de LPA antes de la transfusión y desarrolló su sintomatología durante la transfusión.

El diagnóstico diferencial es sobre todo con la sobrecarga circulatoria causada por la transfusión. Nuestra paciente no tuvo hepatomegalia, plétora yugular, ritmo de galope, ni ninguna otra evidencia clínica de sobrecarga circulatoria; con un valor de péptido natriurético tipo B postransfusional en cifras normales.

El diagnóstico diferencial debe incluir también la reacción anafiláctica, la contaminación bacteriana de los productos hemáticos transfundidos y la reacción hemolítica transfusional. Además se debe tener en cuenta los factores de riesgo 
de SDRA: shock séptico, síndrome de aspiración gástrica, contusión pulmonar, neumonía, sobredosis de fármacos, etc.

El tratamiento de la TRALI es de apoyo con suplementos de oxígeno. Requiere ventilación mecánica hasta en $70 \%$ de los casos graves de TRALI. Los corticoides y los diuréticos se han utilizado, aunque su uso sigue siendo controvertido; pueden ser perjudiciales en el paciente hipovolémico e hipotenso. ${ }^{15}$

Si la TRALI se sospecha durante la transfusión, ésta debe ser suspendida inmediatamente. Se debe notificar dicho evento y devolver el producto sanguíneo restante al banco de sangre, indicando el orden en el que se transfundieron las unidades, para una investigación de la reacción transfusional. Se debe excluir temporalmente de la donación a todos los donadores implicados, hasta que se dispongan de resultados que esclarezcan la causa. ${ }^{16}$

Habitualmente se realiza la búsqueda de anticuerpos con especificidad HLA o contra antígenos específicos de neutrófilos en el suero del donador y en el receptor, aunque el estudio de este último puede obviarse si está recibiendo productos leucorreducidos. Para catalogar el TRALI de inmune es fundamental que los anticuerpos detectados en el donador correspondan con el fenotipo o genotipo del receptor, o bien que la prueba cruzada sea positiva. El hallazgo por sí solo no es suficiente si no se prueba esa correlación.

Como ya se ha comentado, con las pruebas disponibles habitualmente en los Bancos de Sangre hospitalarios no es posible realizar un diagnóstico de laboratorio definitivo de TRALI. Para ello es necesario realizar estudios específicos de anticuerpos y antígenos leucocitarios que habitualmente solo están disponibles en centros de transfusión o de referencia. Estos estudios deberían incluir una prueba cruzada serológica leucocitaria entre el suero del donante y las células del paciente, pero como es una prueba logísticamente difícil de realizar, habitualmente el estudio se inicia con el cribado de anticuerpos HLA y HNA en los donantes; si éstos son positivos, se procede a la identificación de estos anticuerpos y al tipaje específico del paciente para esos antígenos. Aún así, un estudio negativo para todas estas pruebas no descarta que no se trate de un cuadro de TRALI no mediado por anticuerpos.

En cualquier caso, y aunque la sospecha clínica sea alta, hay estudios básicos que siempre se deben llevar a cabo en el laboratorio del banco de sangre hospitalario para descartar que no se trate de alguna de las otras reacciones adversas descritas previamente en el diagnóstico diferencial.

Así, el estudio inmunohematológico eritrocitario en donante y receptor es útil para descartar una reacción hemolítica aguda, la realización de cultivo microbiológico en receptor y producto para descartar bacteriemia por contaminación microbiológica y la realización de un péptido natriurético tipo $B$ para descartar que no se trate de una sobrecarga circulatoria.

En prácticamente todas las comunidades autónomas de nuestro país se realiza desleucotización "universal", por lo que todos los productos transfundidos en nuestro centro están desleucocitados, no estando indicada la realización de anticuerpos antileucocitarios en este caso.

Todos los pacientes que se hayan visto implicados en un caso de TRALI deben recibir productos desleucocitados en caso de que se trate de un centro o país dónde esta medida no se realice de forma sistemática. Por otro lado, se han desarroIlado filtros que absorben anticuerpos y lípidos que podrían mitigar el riesgo de TRALI, pero de momento sólo se ha demostrado su eficacia en 
modelos animales ${ }^{17}$. Si en un futuro se confirmaran estos datos en humanos, también habría que valorar coste beneficio de la implementación de esta medida en la práctica habitual.

No existe un número suficiente de casos publicados de la TRALI en niños para determinar el pronóstico. La lesión pulmonar en pacientes con la TRALI suele ser de duración; mejora la sintomatología en las primeras 72-96 horas, con normalización radiológica en los primeros cuatro días.

La mejor contribución de los pediatras para evitar esta complicación es aplicar una adecuada política transfusional, indicando la transfusión en casos estrictamente necesarios. La prevención de la TRALI se centra en tres estrategias: una política de exclusión de los donantes, una actuación en el almacenamiento y como ya se ha mencionado evitando las transfusiones innecesarias. ${ }^{18}$ Con respecto a la política de exclusión de los donantes se conoce que una gran parte de los casos de TRALI son secundarios a la transfusión de productos hemáticos obtenidos de mujeres multíparas o mujeres con tres o más embarazos. La recomendación de no transfundir plasma procedente de donadoras en diferentes países europeos ha disminuido el número de casos identificados por los sistemas de hemovigilancia y los casos fatales son excepcionales. Algunos autores consideran más razonable la posibilidad de realizar estudio de los donantes con historia de transfusión y/o gestación para anticuerpos anti-HNA y anti-HLA, pero se debe tener en cuenta que son técnicas caras, todavía no disponibles en todos los bancos de sangre y centros de transfusión.

En nuestro caso, uno de los concentrados de hematíes implicados procedía de una mujer multípara encontrándose en el estudio de lookback (investigación retrospectiva de receptores) realizado a la misma la presencia de anticuerpos anti-neutrófilos, probablemente resultado de la exposición durante el embarazo de antígenos leucocitarios paternos.

Otra estrategia de prevención podría ser el acortamiento de los tiempos de almacenamiento de concentrados de hematíes y plaquetas (de especial utilidad para el TRALI no inmune). Debido a que sólo los elementos celulares son capaces de generar lípidos con actividad biológica durante el almacenamiento, se ha pensado que aquellos pacientes con una enfermedad de base que los haría subsidiarios de desarrollar una TRALI no inmune podrían beneficiarse de recibir productos hemáticos "frescos" (p. ej.: concentrados de hematíes de menos de 2 semanas de almacenamiento y plaquetas de menos de 3 días), lo que minimizaría la exposición a lípidos activos capaces de desencadenar el "segundo evento" en los enfermos críticos. El uso de productos sanguíneos lavados para eliminar lípidos activos (sin efectividad demostrada) y la leucorreducción por filtración pre-almacenamiento de todos los productos, que disminuiría la incidencia de TRALI causada por anticuerpos antileucocitarios en el receptor y probablemente reduciría las reacciones de etiología no inmune, serían otras estrategias.

En España la leucorreducción por filtración prealmacenamiento se realiza desde el año 2002 y en México se cuenta con filtros pre almacenamiento y para uso a pie de cama con efecto idéntico.

\section{CONCLUSIÓN}

La TRALI en pacientes pediátricos es una complicación importante de la transfusión de sangre; se debe poner énfasis en la necesidad de cumplir estrictamente las guías transfusionales. Ante la sospecha de un caso, aunque sólo sea probable, hay que evitar la transfusión, remitir la bolsa y muestras del paciente al banco de sangre para el 
estudio pertinente en los donadores implicados y decidir su exclusión como donantes. El informe de estos casos en el sistema de hemovigilancia es fundamental para continuar profundizando en su conocimiento.

\section{REFERENCIAS}

1. Toy P, Popovsky MA, Abraham E, Ambruso DR, Holness LG, Kopko PM, et al. Transfusion-related acute lung injury: definition and review. Crit Care Med. 2005;33:721-726.

2. Food and Drug Administration. Fatalities reported to FDA following blood collection and transfusion: annual summary for fiscal year 2012. 2013. [cited 2013 Sep 7]. Available from: http://www.fda.gov/BiologicsBloodVaccines/SafetyAvailability/ReportaProblem/TransfusionDonationFatalities/ucm346639.htm

3. The Annual SHOT Report 2015. Available at: www.shotuk. org/wp-content/uploads/SHOT-2015-Annual-Report-webEdition-Final-bookmarked.pdf

4. Kleinman S. A perspective on transfusion-related acute lung injury two years after the Canadian Consensus Conference. Transfusion. 2006;46:1465-1468.

5. Mulder HD, Augustijn Q, Van Woensel JB, Bos AP, Juffermans NP, Wösten-Van Asperen RM. Incidence, risk factors, and outcome of transfusion-related acute lung injury in critically ill children: a retrospective study. J Crit Care. 2015;30:55-59.

6. Philipps E, Fleischner FG. Pulmonary edema in the course of a blood transfusion without overloading the circulation. Dis Chest. 1966;50:619-623.

7. Lieberman L, Petraszko T, Yi Q-L, Hannach B, Skeate R. Transfusion-related lung injury in children: a case series and review of the literature. Transfusion. 2014;54:57-64.
8. Weber LL, Roberts LD, Sweeney JD. Residual plasma in red blood cells and transfusion-related acute lung injury. Transfusion. 2014;54:2425-2430.

9. Dotis J, Stabouli S, Violaki A, Mitroudi M, Oikonomou M, Athanassiou-Metaxa M, Kotsiou M. Transfusion-related acute lung injury management in a pediatric intensive care unit. Hippokratia. 2011;15:184-186.

10. Marik PE, Corwin HL. Acute lung injury following to transfusion: Expanding the definition. Crit Care Med. 2008;36:3080-3084.

11. Peter AL, Van Stein D, Vlaar A. Antibody mediated transfusion-related acute lung injury; from discovery to prevention. Br J Haematol 2015;170:597-614.

12. Añón JM, García de Lorenzo A, Quintana M, González E, Bruscas MJ. Lesión pulmonar aguda producida por transfusión. Med Intensiva. 2010;34:139-149.

13. Mazzei CA., Popovsky MA., Kopko PM, 2015. Noninfectious complications of blood transfusion. En: Fung MK., Grossman BJ., Hillyer CD., Westhoff CM., MT (ASCP) SBB. Technical Manual of the American Assoc of Blood Banks. 18th edition. USA. 2015. p. 665-696.

14. Robillard P, Nawej KI, Jochem K. The Quebec hemovigilance system: description and results from the first two years. Transfus Apher Sci. 2004;31:111-122.

15. Church GD, Price C, Sánchez R, Looney MR. Transfusionrelated acute lung injury in the paediatric patient: two case reports and a review of the literature. Transfusion Medicine. 2006;16:343-348.

16. Webert K, Blajchman M. Transfusion-related acute lung injury. CurrOpinHematol. 2005;12:480-487.

17. Silliman CC, Kelher MR, Khan SY, et al. Experimental prestorage filtration removes antibodies and decreases lipids in RBC supernatants mitigating TRALI in vivo. Blood. 2014;123:3488-95

18. Reesink HW, Lee J, Keller A, et al. Measures to prevent transfusion-related acute lung injury (TRALI). Vox Sanguinis. 2012;103:231-259. 